\title{
3D Reconstruction of Tensors and Vectors
}

\author{
Michel Defrise ${ }^{\dagger}$ and Grant T. Gullberg ${ }^{\dagger \dagger}$ \\ $\dagger$ Vrije Universiteit Brussel \\ Brussels, Belgium \\ $\dagger$ E. O. Lawrence Berkeley National Laboratory \\ Berkeley, CA, USA
}

Technical Report LBNL-54936

Lawrence Berkeley National Laboratory

February 22, 2005 


\section{Introduction}

Tensor tomography is a new area of biomedical imaging research which was alluded to in a report from the National Academy of Sciences [1] and builds upon the significant amount of work performed over the last ten years in vector field tomography [2]. Work in this area offers the potential for significant new mathematical developments in the field of inverse problems. The development of new algorithms will have important application to in vivo mapping of brain and spine diffusion tensor fields and cardiac strain-rate and diffusion tensor fields using MRI. Also there will be applications to other imaging modalities involving acoustic or electromagnetic radiation to measure tensor quantities of dielectric, permittivity, conductivity, strain, stress, and elasticity in biological tissues. Measurements of the diffusion tensor field allow to determine the fiber bundle position and orientation in the heart, which can be used to model the mechanical properties of the heart [3]. The electrical conductivity properties in the heart can also be inferred from the diffusion tensor field [4], leading to the possibility for improved modeling of the forward problem [5] for solving the inverse MCG problem [6]. These aspects of the tensor tomography problem present fascinating new areas of applied research in biosciences.

Helmholtz Decomposition for Vector Fields First, to understand the history of vector field tomography development one must understand the Helmholtz decomposition of a vector field. The Helmholtz decomposition states that a vector field can be written as the sum of a solenoidal component (divergence-free, also referred to as a source-free component) and an irrotational component (curl-free component). The solenoidal component can be expressed as the curl of a vector potential $(\nabla \times \Psi)$ and the irrotational component can be expressed as the gradient of a scalar potential $(\nabla \Phi)$. In our discussion to follow, the importance of the Helmholtz decomposition in determining which projection measurements will reconstruct solenoidal components and which will reconstruct irrotational components of the vector field will become clear.

History of Vector Field Tomography Tensor tomography builds on much of the work already accomplished in vector field tomography $[2,7,8,9,10,11,12,13,14,15,16,17,18$, 19, 20, 21, 22, 23, 24, 25, 26, 27, 28, 29, 30, 31, 32, 33, 34, 35]. Applications have involved acoustic flow imaging using time-of-flight measurements in medicine [7], non-destructive evaluation [11], and ocean tomography $[9,14]$. In one of the earliest studies Johnson et al. [7] used ultrasound to reconstruct velocity vector fields in blood vessels from acoustic time-offlight measurements. An iterative algebraic reconstruction technique (ART) was used to compute what was later understood by Norton $[16,21]$ to be the divergence-free component of the vector field. Using the Helmholtz decomposition and the Fourier central-slice theorem, Norton $[16,21]$ derived a reconstruction method for the velocity field and showed that the reconstruction of the acoustic time-of-flight (longitudinal projection [20]) measurements produced only the divergence-free component of the vector field. Before Norton's important contribution, Kramer and Lauterbur [10] developed a hybrid filtered backprojection algorithm to reconstruct flow vector fields using NMR, and also showed that some flow components could not be recovered from only longitudinal projection measurements. Winters and Rouseff [18] developed a Fourier central section theorem for the reconstruction of the divergence-free component, which they argued was important for specifying the vorticity of fluid flow [18, 23, 19]. Later Braun and Hauck [20] showed that projection of the orthogonal 
component of the velocity vector field (the transverse projection measurement) allows one to reconstruct the irrotational component of the vector field. This irrotational (curl-free) component, along with the divergence-free (solenoidal) component, gives the complete determination of the flow vector field. Later, Prince [34] extended the work in 2D to 3D, developing filtered backprojection algorithms for reconstructing both the solenoidal and irrotational components of the vector field from 3D Radon projections. Prince generalized the longitudinal and transverse measurements of Braun and Hauck [20] by defining a more general inner product measurement (probe transform), which forms an inner product between the vector field and a unit-vector probe direction. Prince [34] showed in 3D that only one set of probe measurements (the irrotational measurements) is required to reconstruct the irrotational component and two sets of probe measurements (the solenoidal measurements) are required to reconstruct the solenoidal component. The three probe directions are such that they form a linearly independent set of vectors.

Vector Field Tomography on Bounded Domains The vector field tomography problem has some interesting characteristics when restricted to bounded domains. In fact, physical problems are often defined on bounded domains and it is the boundary that creates or partially defines the field. Norton [16, 21], Braun and Hauck [20], and Osman and Prince [35] were all concerned about the vector field tomography problem on bounded domains. Norton showed that the longitudinal measurements with boundary conditions reconstruct a divergence-free vector field composed of a solenoidal component that satisfies homogeneous boundary conditions and an irrotational component defined by the gradient on the boundary. It was recognized by Braun and Hauck [20] that bounded domains admit harmonic vector fields that are both irrotational and solenoidal. Therefore, the decomposition into irrotational and solenoidal components is not unique. They proposed that the decomposition should include irrotational homogeneous, solenoidal homogeneous, and harmonic components where the divergence and curl of the harmonic component is always zero. The solenoidal component is homogeneous in that its normal component is zero on the boundary (completely tangential). The curl-free component is homogenous in that its tangential component is zero on the boundary (totally normal). Braun and Hauck [20] showed that the harmonic component of a $2 \mathrm{D}$ vector field is reconstructed equally between the irrotational and solenoidal measurements, whereas Osman and Prince [35] showed that the harmonic component of a $3 \mathrm{D}$ vector field is not imaged equally in the irrotational and solenoidal measurements. They characterized the homogenous component as a gradient of a harmonic function satisfying Laplace's equation. They showed how one can separate homogeneous and harmonic components from reconstruction of solenoidal and irrotational components of the vector field.

Other Works Vector field tomography has been applied in other applications: optics, to measure flow $[16,17,21]$; deflection optical tomography, to determine densities in supersonic expansions and flames [15]; optical polarization tomography, to measure electric fields in a Kerr material by measuring polarization of the transmitted light [12, 13, 25]; acoustic tomography, to determine temperature distributions and velocity vector fields in furnaces [26]; polarimetric tomography of the magnetic field in TOKOMAK[8, 27]; and velocity vector fields of heavy particles in plasma $[32,28]$. It has also been shown that continuous doppler data can be analyzed in the framework of vector field tomography [2, 21, 24, 22, 33, 32, 28, 29]. History of Tensor Field Tomography As seen in the previous discussion, vector field 
tomography has been an active area of research over the last decade for several applications, including medical imaging. Originally, the most active area of application of tensor tomography was photoelasticity $[36,37,38,39,40,41,42,43]$ and that continues to be so $[44,45,46,47,48,49,50]$. An early book on the subject of photoelasticity was written by Aben [36]. This application stimulated Sharafutdinov [51] to write a monograph on the mathematical theory of integral geometry of nth-order tensor fields. A most interesting result presented in this monograph is that a symmetric tensor field also has a Helmholtz type decomposition in that it can be decomposed into a sum of a divergence-free component and a curl-free component.

There has been little work besides ours $[52,53,54,55,56,57,58,59,60]$ in the field of medical imaging. In our initial work an algorithm was developed for the reconstruction of 2D tensor fields of second order tensors [52]. This algorithm was verified in computer simulations and applied to the reconstruction of 2D diffusion tensor fields from MRI tensor projections. The 2D reconstruction algorithm was extended to 3D $[53,56]$. Both filtered backprojection [56] and backprojection filtering [54] approaches to reconstruction of 3D tensor fields from 3D planar (Radon) and line-integral (X-ray) tensor projections were formulated. In [56] a Fourier projection theorem for Radon projections of 3D second order tensor fields was developed. We also developed iterative algorithms for solving the nonlinear diffusion tensor MRI tomographic problem $[55,57,58]$. We applied this algorithm to reconstruct only the principal component of the diffusion tensor [58]. We also proposed the application of tensor tomography as a more efficient method of obtaining diffusion tensor fields with MRI using the PROPELLER pulse sequence [60]. We expect that all MRI tensor tomography data will be processed with an iterative reconstruction algorithm to avoid any inaccuracies as the result of linearizing the model which is required in the application of any linear tomographic reconstruction algorithms.

Extension of Our Results to Physical and Mathematical Sciences We believe the work presented in this report can lead to tensor reconstruction work in not only diffusion tensor magnetic resonance imaging (DT-MRI) but in various other physical and mathematical sciences. Most closely related is diffraction tomography (DTM) of acoustical media [61]. In fact, DTM and CT reconstruction of vector fields closely parallel each other. It can be assumed, therefore, that the methods outlined in this report can be used in reconstructing the 'kinetic tensor' [62] in fluids. This is important in studying fluids with suspensions and fogs. This may also result in clear-air turbulence prediction which is vital to aviation safety. This applies also to elastic media under the Born approximation [63, 64]. The so-called 'pitch-catch' technique for recovering the Lam parameters was a rudimentary attempt at using a tensor field 'probe' (see [34] for definition of probe). Tensor tomography offers the potential for more systematic studies. Also, in recent years inverse source problems involving the 'kinetic equation' (Boltzman equation with the collision integral) have attracted much attention [65]. This equation governs wide-ranging physical problems involving transfer of radiation (plasmas, magnetohydrodynamics, astrophysics, and so forth). Since the exact collision integral is not tractable, it is customary to use the well-known Fokker-Planck approximation [66] which results in a diffusion tensor term that arises out of velocity-exchanges. In addition, there are other areas of physics where the results of this report may prove to be stimulating. These include nonlinear optics (reconstruction of the susceptibility tensor); anisotropic electromagnetic $[67,68]$ and elastic media (the latter being of significant current interest); stress tensor 
polarization tomography in photo- and magnetoelastic materials which are already being pursued vigorously [41, 42, 45, 46, 47, 48, 49, 50]; second and fourth-order tensor tomography from seismic traveltime tomographic data [69]; neutron spin tensor tomography [70]; and general relativity tensor tomography using gravitational delay time tomographic data [71]. Tomography also can be applied to finding inverse solutions of integral transforms of differential forms [72] or finding a symmetric tensor field from its integrals over all geodesics of a Riemannian metric [51].

This report presents some of the mathematical results of vector and tensor tomography. An important result is the development of Fourier projection theorems for the decomposition of vector and tensor fields into a sum of a divergence-free component and a curl-free component. The results apply to the linear vector and tensor tomography problem. However, the mathematics provides a structure for understanding the data sampling requirements for the nonlinear tensor tomography problem posed by diffusion tensor MRI.

\section{The 3D X-ray transform and 3D Radon transform of vectors and tensors: definitions}

\subsection{Vectors and tensors}

A vector field in $\mathbb{R}^{3}$ will be denoted by its three components $v_{i}(x), i=1,2,3, x \in \mathbb{R}^{3}$ along the $x, y$ and $z$ axis respectively. We assume that each component is a smooth function with a compact support. Similarly, we consider symmetric tensor fields denoted by their components $t_{i, j}(x)=t_{j, i}(x), i, j=1,2,3$. Again, we assume that each component is smooth and has a compact support. The Fourier transform of a tensor or vector is defined by taking the usual Fourier transform of each component, e.g.,

$$
\hat{t}_{i, j}(\nu)=\int_{\mathbb{R}^{3}} d x \exp (-2 \pi i x \cdot \nu) t_{i, j}(x) \quad \nu \in \mathbb{R}^{3} .
$$

\subsection{The 3D X-ray transform: directional measurements}

The 3D X-ray transform is normally defined for scalar functions. Projection imaging of a vector or tensor field involves measuring the usual 3D X-ray transform of their scalar product with some unit vector, thus defining a scalar directional measurement. A well-known example is the vectorial X-ray transform of a vector field, which is the quantity measured using Doppler imaging of velocity fields. In this case, one measures the X-ray transform of the scalar product of the velocity with the unit vector $\theta$ along the rays:

$$
p^{\theta}(s, \theta)=\int_{-\infty}^{+\infty} d l \theta_{j} v_{j}(s+l \theta) \quad s \in \theta^{\perp}, \theta \in \Omega,
$$

where $\Omega \subset S^{2}$ is the subset of directions for which parallel projections are measured. Note the use of the convention that summation is taken over repeated indices, i.e.

$$
\theta_{j} v_{j} \equiv \sum_{j=1}^{3} \theta_{j} v_{j}=\theta \cdot v \text {. }
$$


More generally, we can define a directional X-ray transform for an arbitrary unit vector $e$ :

$$
p^{e}(s, \theta)=\int_{-\infty}^{+\infty} d l e_{j} v_{j}(s+l \theta)
$$

Intuitively, it is obvious that three independent directional measurements are required to recover the $3 \mathrm{D}$ vector field $v$. We could for instance use the three axis, $e=1_{x}, e=$ $1_{y}$ and $e=1_{z}$, which would amount to measuring separately the X-ray transform of the three components of the vector (that is, the component-wise X-ray transform). In view of the example in Doppler imaging, it is often more natural to consider more general sets of directional data defined using unit vectors which may depend on the direction $\theta$ of the ray. These vectors will be denoted $e^{\alpha}(\theta), \alpha=1,2,3$, or simply $e^{\alpha}$. In section 5 , specific results will be obtained for the case where $e^{1}=\theta$, and $e^{2}$ and $e^{3}$ are in the plane $\theta^{\perp}$ orthogonal to $\theta$. Here we only assume that the vectors $e^{\alpha}(\theta), \alpha=1,2,3$ are orthonormal for each direction $\theta$, and we discuss the reconstruction of a vector field $v(x)=\left(v_{1}(x), v_{2}(x), v_{3}(x)\right)$ from the three directional X-ray transforms:

$$
p^{e^{\alpha}}(s, \theta)=\int_{-\infty}^{+\infty} d l e_{j}^{\alpha}(\theta) v_{j}(s+l \theta) .
$$

To shorten notations, this will be written $p^{\alpha}$ and the $\theta$ dependence of the unit vectors $e^{\alpha}$ will be omitted.

The previous discussion can be generalized to a tensor. In this case the tensor must be contracted with two unit vectors to get a scalar function which can be integrated along lines, and we have therefore nine directional measurements (six for a symmetric tensor) defined by

$$
p^{e^{\alpha}, e^{\beta}}(s, \theta)=\int_{-\infty}^{+\infty} d l e_{i}^{\alpha} t_{i, j}(s+l \theta) e_{j}^{\beta} \quad \alpha, \beta=1,2,3
$$

(again noted $p^{\alpha, \beta}$ for simplicity).

An important remark, both for vectors and tensors, is that a single directional measurement defined by equation (5) or (6) is not the 3D X-ray transform of some scalar function. This is because the scalar product is taken with a vector $e^{\alpha}$ that depends on the direction of projection $\theta$, and therefore the data for various directions $\theta$ will not in general be consistent. For instance the simplest consistency condition for the X-ray transform is that the integral of a $2 \mathrm{D}$ parallel projection must be independent of $\theta$. This condition is not satisfied in our case since one easily checks that:

$$
\int_{\theta^{\perp}} d s p^{\alpha}(s, \theta)=e_{j}^{\alpha}(\theta) \int_{\mathbb{R}^{3}} d x v_{j}(x)
$$

depends on $\theta$ through $e^{\alpha}$. This also holds for tensors.

\subsection{The 3D Radon transform: directional measurements}

The 3D Radon transforms of vectors and tensors are defined as for the 3D X-ray transform, using the same directional measurements defined by the orthonormal vectors $e^{1}, e^{2}$ and $e^{3}$. In this case, $\theta$ is the normal to the plane on which integration is performed. The directional measurements are then defined as: 


$$
r^{\alpha}(s, \theta)=\int_{\mathbb{R}^{3}} d x e_{j}^{\alpha} v_{j}(x) \delta(x \cdot \theta-s) \quad s \in \mathbb{R}, \theta \in S^{2}, \alpha=1,2,3
$$

for vectors. When $e^{1}=\theta$ as in section $5, r^{1}$ is called the normal Radon transform by Sparr [2]. For tensors, similarly:

$$
r^{\alpha, \beta}(s, \theta)=\int_{\mathbb{R}^{3}} d x e_{i}^{\alpha} t_{i, j}(x) e_{j}^{\beta} \delta(x \cdot \theta-s) \quad s \in \mathbb{R}, \theta \in S^{2}, \alpha, \beta=1,2,3 .
$$

\section{Central section theorems and directional backpro- jection}

\subsection{D X-ray transform}

A key property of the X-ray transform is the central section theorem. This theorem is easily extended by noting that each directional measurement is simply the scalar product of the vector $e^{\alpha}$ with the component wise transform of the vector or tensor. This leads to:

$$
\hat{p}^{\alpha}(\nu, \theta)=e_{i}^{\alpha} \hat{v}_{i}(\nu) \quad \nu \cdot \theta=0,
$$

and

$$
\hat{p}^{\alpha, \beta}(\nu, \theta)=e_{i}^{\alpha} \hat{t}_{i, j}(\nu) e_{j}^{\beta} \quad \nu \cdot \theta=0,
$$

where the Fourier transforms are with respect to the non-angular variables, e.g.,

$$
\hat{p}^{\alpha}(\nu, \theta)=\int_{\theta^{\perp}} d s p^{\alpha}(s, \theta) \exp (-2 \pi i s \cdot \nu) \quad \nu \cdot \theta=0 .
$$

Let us now define the backprojection operation. Since a given directional measurement equation $(5,6)$ maps a vector (tensor) onto a scalar function, the backprojection, which is the dual operation, must map a scalar data function onto a vector (tensor). One checks that the appropriate backprojections must be defined as:

$$
b_{i}^{\alpha}(x)=\int_{\Omega} d \theta p^{\alpha}(x-(x \cdot \theta) \theta, \theta) e_{i}^{\alpha}(\theta) \quad \alpha=1,2,3,
$$

and

$$
b_{i, j}^{\alpha, \beta}(x)=\int_{\Omega} d \theta p^{\alpha, \beta}(x-(x \cdot \theta) \theta, \theta) e_{i}^{\alpha}(\theta) e_{j}^{\beta}(\theta) \quad \alpha, \beta=1,2,3 .
$$

Note that the vectors $e^{\alpha}$ depend on the direction $\theta$ and hence these backprojections have angular weights and do not coincide with the usual scalar backprojection.

The 3D Fourier transform of the backprojection of a directional measurement $\alpha$ (or $\alpha, \beta)$ can be calculated using the central section theorem above. For instance, one has for vectors: 


$$
\begin{aligned}
\hat{b}_{i}^{\alpha}(\nu) & =\int_{\mathbb{R}^{3}} d x \exp (-2 \pi i x \cdot \nu) b_{i}^{\alpha}(x) \\
& =\int_{\Omega} d \theta e_{i}^{\alpha} \int_{\theta^{\perp}} d s \exp (-2 \pi i s \cdot \nu) p^{\alpha}(s, \theta) \int_{\mathbb{R}} d l \exp (-2 \pi i l \nu \cdot \theta) \\
& =\int_{\Omega} d \theta e_{i}^{\alpha} \hat{p}^{\alpha}(\nu, \theta) \delta(\nu \cdot \theta) \\
& =\frac{1}{\|\nu\|} \hat{v}_{j}(\nu) \hat{k}_{i, j}^{\alpha}\left(\frac{\nu}{\|\nu\|}\right) \quad \nu \in \mathbb{R}^{3},
\end{aligned}
$$

where we have for each direction $\theta$ decomposed the position vector as $x=s+l \theta$ with $s \cdot \theta=0$, and the filter $\hat{k}$ is a tensor given by

$$
\hat{k}_{i, j}^{\alpha}(n)=\int_{\Omega} d \theta e_{i}^{\alpha}(\theta) e_{j}^{\alpha}(\theta) \delta(n \cdot \theta) \quad n \in S^{2} .
$$

For tensors one finds similarly:

$$
\hat{b}_{i, j}^{\alpha, \beta}(\nu)=\frac{1}{\|\nu\|} \hat{t}_{k, l}(\nu) \hat{k}_{i, j, k, l}^{\alpha, \beta}\left(\frac{\nu}{\|\nu\|}\right)
$$

with a contraction over the indices $k$ and $l$. The filter is here a tensor with four indices:

$$
\hat{k}_{i, j, k, l}^{\alpha, \beta}(n)=\int_{\Omega} d \theta e_{i}^{\alpha}(\theta) e_{j}^{\beta}(\theta) e_{k}^{\alpha}(\theta) e_{l}^{\beta}(\theta) \delta(n \cdot \theta) .
$$

\subsection{D Radon transform}

The central section theorem for the directional Radon transform is easily found to be:

$$
\hat{r}^{\alpha}(\nu, \theta)=e_{j}^{\alpha} \hat{v}_{j}(\nu \theta) \quad \nu \in \mathbb{R}, \alpha=1,2,3,
$$

and for tensors

$$
\hat{r}^{\alpha, \beta}(\nu, \theta)=e_{i}^{\alpha} \hat{t}_{i, j}(\nu \theta) e_{j}^{\beta} \quad \nu \in \mathbb{R}, \alpha, \beta=1,2,3 .
$$

The dual operator is here also a directional backprojection involving an angular weight defined by the vectors $e^{\alpha}(\theta)$. These operator map each directional measurement onto a vector (tensor) as follows:

or

$$
b_{i}^{\alpha}(x)=\int_{S^{2}} d \theta r^{\alpha}(x \cdot \theta, \theta) e_{i}^{\alpha}
$$

$$
b_{i, j}^{\alpha, \beta}(x)=\int_{S^{2}} d \theta r^{\alpha}(x \cdot \theta, \theta) e_{i}^{\alpha} e_{j}^{\beta} .
$$

The 3D Fourier transform of these backprojections have again a simple relation with the original vector or tensor, namely, with $n=\nu /\|\nu\|$,

or

$$
\hat{b}_{i}^{\alpha}(\nu)=\frac{2}{\|\nu\|^{2}} e_{i}^{\alpha}(n) e_{j}^{\alpha}(n) \hat{v}_{j}(\nu)
$$

$$
\hat{b}_{i, j}^{\alpha, \beta}(\nu)=\frac{2}{\|\nu\|^{2}} e_{i}^{\alpha}(n) e_{j}^{\beta}(n) e_{k}^{\alpha}(n) e_{l}^{\beta}(n) \hat{t}_{k, l}(\nu) \text {. }
$$




\section{Reconstruction from the complete set of directional data}

\subsection{The X-ray transform}

When the whole set of directional data is available the vector or tensor field can be reconstructed easily using standard filtered backprojection. Indeed, using the completeness relation

$$
\sum_{\alpha=1}^{3} e_{i}^{\alpha} e_{j}^{\alpha}=\delta_{i, j}
$$

which holds for any triple of orthonormal basis vectors, one can recover the 3D X-ray transform of any component of the vector (tensor) as a linear combination of the directional measurements. For example,

$$
\sum_{\alpha=1}^{3} e_{i}^{\alpha} p^{\alpha}(s, \theta)=\int_{-\infty}^{+\infty} d l \sum_{\alpha=1}^{3} e_{i}^{\alpha} e_{j}^{\alpha} v_{j}(s+l \theta)=\int_{-\infty}^{+\infty} d l \delta_{i, j} v_{j}(s+l \theta)=\int_{-\infty}^{+\infty} d l v_{i}(s+l \theta)
$$

is the X-ray transform of the function $v_{i}(x)$. Applying usual 3D filtered-backprojection one obtains:

$$
v_{i}(x)=\sum_{\alpha=1}^{3} \int_{\Omega} d \theta p^{F \alpha}(x-(x \cdot \theta) \theta, \theta) e_{i}^{\alpha},
$$

with filtered data given by

$$
p^{F \alpha}(s, \theta)=\int_{\theta^{\perp}} d s^{\prime} h_{\Omega}\left(s-s^{\prime}\right) p^{\alpha}\left(s^{\prime}, \theta\right),
$$

and $h_{\Omega}$ is the kernel associated with the Colsher filter [73] corresponding to the set of measured directions $\Omega$ ( assumed to satisfy Orlov's condition [74] ):

$$
h_{\Omega}(s)=\int_{\mathbb{R}^{3}} d \nu \exp (2 \pi i \nu \cdot s) \hat{h}_{\Omega}(\nu)
$$

with Colsher's filter

$$
\hat{h}_{\Omega}(\nu)=\frac{1}{\int_{\Omega} d \theta \delta(\nu \cdot \theta)}=\frac{\|\nu\|}{\int_{\Omega} d \theta \delta(\nu \cdot \theta /\|\nu\|)} .
$$

Thus reconstruction from a complete set of directional data is a straightforward extension of the standard scalar inversion. Note however that the backprojection in equation (13) has an angular weight introduced by $e_{i}^{\alpha}$.

For tensor the same approach yields

$$
t_{i, j}(x)=\sum_{\alpha=1}^{3} \sum_{\beta=1}^{3} \int_{\Omega} d \theta p^{F \alpha, \beta}(x-(x \cdot \theta) \theta, \theta) e_{i}^{\alpha} e_{j}^{\beta} .
$$

An alternative consists in first applying the directional backprojection as in equation (13) and (14), and then using the completeness relation equation (25). After taking the 3D Fourier transform, one has:

$$
\sum_{\alpha=1}^{3} \hat{b}_{i}^{\alpha}(\nu)=\frac{1}{\|\nu\|} \hat{v}_{j}(\nu) \sum_{\alpha=1}^{3} \hat{k}_{i, j}^{\alpha}\left(\frac{\nu}{\|\nu\|}\right) .
$$


But from equations (16) and (25)

$$
\sum_{\alpha=1}^{3} \hat{k}_{i, j}^{\alpha}(n)=\delta_{i, j} \int_{\Omega} d \theta \delta(n \cdot \theta) \quad n \in S^{2}
$$

which is the product of the Kronecker tensor with the reciprocal of the angular part of Colsher's filter. Finally, reconstruction reads:

$$
\hat{v}_{i}(\nu)=\hat{h}_{\Omega}(\nu) \sum_{\alpha=1}^{3} \hat{b}_{i}^{\alpha}(\nu) .
$$

Reconstruction is simple: sum the directional backprojections of the three data $\alpha=1,2,3$ and filter the result using the usual 3D Colsher filter. A similar result holds for tensors.

To conclude this section, we give for completeness the expression of Colsher filter for the case where the set of measured directions is an equatorial band with axis $a=(0,0,1)$ and aperture $2 \theta_{0}$, i.e. $\Omega=\left\{u \in S^{2}:\left|u_{z}\right| \leq \sin \theta_{0}\right\}$ :

$$
\begin{aligned}
\left(\hat{h}_{\Omega}(n)\right)^{-1} & =4 \arcsin \left(\frac{\sin \theta_{0}}{|\sin \psi|}\right) & & |\sin \psi|>\sin \theta_{0} \\
& =2 \pi & & |\sin \psi| \leq \sin \theta_{0}
\end{aligned}
$$

where $\sin \psi=\sqrt{1-n_{z}^{2}}$.

\subsection{The Radon transform}

Using the same completeness relation in equation (25), the usual filtered-backprojection inversion of the 3D X-ray transform can be extended to vectors and tensors when all directional measurements are available. Following the same lines as above, one finds for vectors:

$$
v_{i}(x)=\frac{-1}{8 \pi^{2}} \sum_{\alpha=1}^{3} \int_{S^{2}} d \theta e_{i}^{\alpha} \frac{\partial^{2}}{\partial s^{2}} r^{\alpha}(s=x \cdot \theta, \theta) .
$$

For tensors,

$$
t_{i, j}(x)=\frac{-1}{8 \pi^{2}} \sum_{\alpha=1}^{3} \sum_{\beta=1}^{3} \int_{S^{2}} d \theta e_{i}^{\alpha} e_{j}^{\beta} \frac{\partial^{2}}{\partial s^{2}} r^{\alpha, \beta}(x \cdot \theta, \theta) .
$$

Reconstruction by filtering the backprojected data can also be obtained from equations (21) and (22).

\section{Reconstruction from a single directional measure- ment}

We have seen in the previous section that the reconstruction of a tensor or vector field from a complete set of directional data is a fairly trivial extension of the usual scalar reconstruction methods. Especially for tensors however, the acquisition of a full set of measurements (i.e 
six measurements $\alpha, \beta)$ may be problematic. The question then naturally arises: which information can be obtained from a smaller set of data? Motivated by the example of the Doppler tomography of velocity field, we focus here on the case where a single directional data is available, that corresponds to the vector $e^{1}(\theta)=\theta$. This means, for the X-ray transform, that we only measure the scalar product of the vector (or tensor) with the unit vector along the integration ray. For the 3D Radon transform, this corresponds to measuring plane integrals of the scalar product with the vector orthogonal to the plane.

Considering first the X-ray transform, we rederive the general result demonstrated in the book by Sharafutdinov [51], namely that it is possible to reconstruct the solenoid part of the vector (or tensor, see below) from that single measurement. We will give a simpler proof of this result for the special case analyzed here, and furthermore generalize this result to the case where the set $\Omega$ of measured projections is smaller than $S^{2}$. In the case of vector fields, this situation was analyzed by Sparr [2].

\subsection{Decomposition of a tensor or vector field into solenoid and potential parts}

As shown in Sharafutdinov, a smooth symmetric tensor field which vanishes sufficiently rapidly at infinity can be decomposed in a unique way as follows:

$$
t_{i, j}(x)={ }^{s} t_{i, j}(x)+\frac{1}{2}\left(\partial_{i} \phi_{j}(x)+\partial_{j} \phi_{i}(x)\right),
$$

where $\phi$ is a vector potential and ${ }^{s} t$ is a symmetric solenoid tensor field, that is one that is divergence free:

$$
\partial_{i}{ }^{s} t_{i, j}(x)=\partial_{j}{ }^{s} t_{i, j}(x)=0 \quad i=1,2,3,
$$

with the usual implicit sum over $j$. This generalises the well-known decomposition of a vector field due to Helmholz:

$$
v_{i}(x)={ }^{s} v_{i}(x)+\partial_{i} \phi(x),
$$

where $\phi$ is a scalar potential and ${ }^{s} v$ is a solenoid vector field, that is one that is divergence free. Usually ${ }^{s} v$ is written as the curl of another divergence free vector field (the vector potential) but we will not use this representation here.

The decomposition into solenoid and potential parts can also be illustrated by considering the Fourier transforms. Noting that the partial derivative with respect to $x_{j}$ is equivalent to the multiplication of the Fourier transform by $-2 \pi i \nu_{j}$, one sees that the divergence free condition for the solenoid component of the tensor becomes:

$$
\nu_{j}{ }^{s} \hat{t}_{i, j}(\nu)=0 \quad i=1,2,3 .
$$

Thus the decomposition amounts to separating the components, in Fourier space, that are parallel to the frequency $\nu$. The solenoid component is then given simply by projecting the Fourier transform of the vector (tensor) onto the plane orthogonal to $\nu[51]$ :

$$
\begin{aligned}
{ }^{s} \hat{v}_{i}(\nu) & =\hat{v}_{i}(\nu)-n_{i}\left(n_{k} \hat{v}_{k}(\nu)\right) \\
{ }^{s} \hat{t}_{i, j}(\nu) & =\hat{t}_{i, j}(\nu)-n_{i}\left(n_{k} \hat{t}_{k, j}(\nu)\right)-n_{j}\left(n_{k} \hat{t}_{i, k}(\nu)\right)+n_{i} n_{j}\left(n_{k} n_{l} \hat{t}_{k, l}(\nu)\right)
\end{aligned}
$$


where $n=\nu /\|\nu\|$. It also follows that the solenoid components of a vector (tensor) field depends on two (three) scalar functions.

We are now going to show, following Sharafutdinov, that the whole solenoid component can be recovered from a single directional measurement, namely $p^{1}$ (for vectors) or $p^{11}$, for tensors. That this is possible at all is due to the redundancy of the 3D X-ray transform.

\subsection{The vector case with full acceptance}

We start with the case where all parallel projections are measured, i.e. $\Omega=S^{2}$ and will use the symmetry properties of the problem to determine the explicit form of the tensor

$$
\hat{k}_{i, j}^{1}(n)=\int_{S^{2}} d \theta \theta_{i} \theta_{j} \delta(n \cdot \theta) \quad n \in S^{2}
$$

in equation (15). To determine the closed form expression of this tensor, we use the a priori knowledge that it can only be a function of the unit vector $n=\nu /\|\nu\|$ since no other specific direction appears in the problem owing to the full rotational symmetry of the set $\Omega$. Hence $\hat{k}_{i, j}$ can only be constructed from $n_{i}$ and from the Kronecker tensor $\delta_{i, j}$ (remember that it is symmetric so we cannot use $\epsilon_{i, j, k}$ ). Thus, there must be constants $A$ and $B$ such that

$$
\hat{k}_{i, j}^{1}(n)=A n_{i} n_{j}+B \delta_{i, j} .
$$

But we also know that our tensor must be divergence free (as can be seen from equation (43)) and therefore we have the additional constraint

$$
n_{i} \hat{k}_{i, j}^{1}(n)=(A+B) n_{j}=0 \rightarrow B=-A .
$$

Finally, by noting that

$$
\hat{k}_{i, i}^{1}(n)=\int_{S^{2}} d \theta \delta(n \cdot \theta)=2 \pi=A+3 B=-2 A,
$$

one finds

$$
\hat{k}_{i, j}^{1}(n)=\pi\left(\delta_{i, j}-n_{i} n_{j}\right) \equiv \pi \epsilon_{i, j}
$$

We are now ready to reconstruct the Fourier transform of the solenoid component of the vector field from the backprojection of the directional measurement $p^{1}$. Indeed, using equation (15) and (47), we have

$$
\begin{aligned}
\hat{b}_{i}^{1}(\nu) & =\frac{1}{\|\nu\|} \hat{v}_{j}(\nu) \hat{k}_{i, j}^{1}\left(\frac{\nu}{\|\nu\|}\right) \\
& =\frac{\pi}{\|\nu\|}\left(\hat{v}_{i}(\nu)-\frac{1}{\|\nu\|^{2}} \nu_{i} \nu_{j} \hat{v}_{j}(\nu)\right),
\end{aligned}
$$

which is the projection of the Fourier transformed vector field onto the plane orthogonal to $\nu$, i.e. the solenoid component of the vector field. One can therefore reconstruct the solenoid part as

$$
{ }^{s} \hat{v}_{j}(\nu)=\frac{\|\nu\|}{\pi} \hat{b}_{i}^{1}(\nu)
$$

Note that, as is well known, the potential part of the vector field is transparent to this directional measurement (i.e. it belongs to its null space). 


\subsection{The tensor case with full acceptance}

We now follow the same lines as above to reconstruct the solenoid component of a symmetric tensor from the directional X-ray transform $p^{11}$, assuming again that $\Omega=S^{2}$ and $e^{1}(\theta)=\theta$. The first step is to determine the explicit form of the tensor $\hat{k}_{i, j, k, l}^{1,1}(n)$ in equation (18). We know that this tensor:

1. is symmetrical for all permutations of its indices,

2. is divergence free, i.e. $n_{i} \hat{k}_{i, j, k, l}^{1,1}(n)=0$,

3. must be built only from $n_{i}$ and from $\delta_{i, j}$.

Using the tensor $\epsilon_{i, j}=\delta_{i, j}-n_{i} n_{j}$, the most general symmetrical form is then

$$
\begin{aligned}
\hat{k}_{i, j, k, l}^{1,1}(n) & =A\left(\delta_{i, j} \delta_{k, l}+\delta_{i, k} \delta_{j, l}+\delta_{i, l} \delta_{j, k}\right) \\
& +B\left(\delta_{i, j} \epsilon_{k, l}+\delta_{i, k} \epsilon_{j, l}+\delta_{i, l} \epsilon_{j, k}+\delta_{k, l} \epsilon_{i, j}+\delta_{j, l} \epsilon_{i, k}+\delta_{j, k} \epsilon_{i, l}\right) \\
& +C\left(\epsilon_{i, j} \epsilon_{k, l}+\epsilon_{i, k} \epsilon_{j, l}+\epsilon_{i, l} \epsilon_{j, k}\right) .
\end{aligned}
$$

Now the condition 2. above implies:

$$
\begin{gathered}
n_{i} n_{j} n_{k} \hat{k}_{i, j, k, l}^{1,1}(n)=0=3 A n_{l} \rightarrow A=0, \\
n_{i} n_{j} \hat{k}_{i, j, k, l}^{1,1}(n)=0=B \epsilon_{k, l} \rightarrow B=0, \\
\hat{k}_{i, j, i, j}^{1,1}(n)=8 C=\int_{S^{2}} d \theta \delta(\theta \cdot n)=2 \pi,
\end{gathered}
$$

and we obtain therefore

$$
\hat{k}_{i, j, k, l}^{1,1}(n)=\frac{\pi}{4}\left(\epsilon_{i, j} \epsilon_{k, l}+\epsilon_{i, k} \epsilon_{j, l}+\epsilon_{i, l} \epsilon_{j, k}\right) .
$$

Consider now the Fourier transform of the backprojection of the directional measurement, which is given by equation (17):

$$
\hat{b}_{i, j}^{1,1}(\nu)=\frac{1}{\|\nu\|} \hat{t}_{k, l}(\nu) \hat{k}_{i, j, k, l}^{1,1}\left(\frac{\nu}{\|\nu\|}\right) .
$$

Using the fact that $\hat{k}^{1,1}$ is divergence free and that

$$
{ }^{s} \hat{t}_{k, l} n_{l}=0 \rightarrow{ }^{s} \hat{t}_{k, l} \epsilon_{l, j}={ }^{s} \hat{t}_{k, j}
$$

only the solenoid component of the tensor $t$ contributes to this measurement, we get:

$$
\hat{b}_{i, j}^{1,1}(\nu)=\frac{\pi}{4\|\nu\|}\left\{\left({ }^{s} \hat{t}_{k, l}(\nu) \delta_{k, l}\right) \epsilon_{i, j}+2^{s} \hat{t}_{i, j}\right\}
$$

Denoting the trace of the solenoid tensor as $\tau(\nu)={ }^{s} \hat{t}_{k, k}(\nu)$, the solenoid part can be reconstructed as:

$$
s \hat{t}_{i, j}(\nu)=\frac{2\|\nu\|}{\pi} \hat{b}_{i, j}^{1,1}(\nu)-\frac{\tau(\nu)}{2} \epsilon_{i, j}
$$


The scalar trace can be determined by noting that

$$
\hat{b}_{k, k}^{1,1}(\nu)=\frac{\pi}{4\|\nu\|}(2 \tau(\nu)+2 \tau(\nu)) \text {. }
$$

The final result demonstrates that the solenoid part of the tensor, which depends on three scalar functions, can be recovered from a single directional measurement of the 3D X-ray transform provided $\Omega=S^{2}$ ([51], theorem 2.12.2) :

$$
{ }^{s} \hat{t}_{i, j}(\nu)=\frac{2\|\nu\|}{\pi}\left\{\hat{b}_{i, j}^{1,1}(\nu)-\frac{1}{4} \hat{b}_{k, k}^{1,1}(\nu)\left(\delta_{i, j}-\frac{\nu_{i} \nu_{j}}{\|\nu\|^{2}}\right)\right\} .
$$

\subsection{The vector case with limited acceptance}

We now show how to analyse the case where the subset of measured parallel projections is limited to an equatorial band $\Omega$ with some fixed symmetry axis $a \in S^{2}$.

The main problem is to build the tensor $\hat{k}_{i, j}^{1}(n)$. In this case, the tensor can be constructed not only from Kronecker's $\delta_{i, j}$ and from the frequency $\nu$, but also from the unit vector $a$ since the set $\Omega$ has only a radial symmetry around this axis, whereas in section 5.1 it had full spherical symmetry. Using the symmetry of the tensor and the fact that it is divergence free, one then obtains the most general expression as $(n=\nu /\|\nu\|)$ :

$$
\hat{k}_{i, j}^{1}(n)=A_{\Omega}(n)\left\{a_{i}-(a \cdot n) n_{i}\right\}\left\{a_{j}-(a \cdot n) n_{j}\right\}+B_{\Omega}(n)\left\{\delta_{i, j}-n_{i} n_{j}\right\} .
$$

To evaluate the functions $A_{\Omega}$ and $B_{\Omega}$, we need two equations which are obtained by contracting the tensor as follows:

$$
\begin{aligned}
J_{\Omega}(n) & \equiv \int_{\Omega} d \theta \delta(n \cdot \theta)=\hat{k}_{i, i}^{1}(n)=A_{\Omega}\left(1-(a \cdot n)^{2}\right)+2 B_{\Omega} \\
J_{\Omega}^{a}(n) & \equiv \frac{1}{1-(a \cdot n)^{2}} \int_{\Omega} d \theta(a \cdot \theta)^{2} \delta(n \cdot \theta) \\
& =\frac{1}{1-(a \cdot n)^{2}} \hat{k}_{i, j}^{1}(n) a_{i} a_{j}=A_{\Omega}\left(1-(a \cdot n)^{2}\right)+B_{\Omega},
\end{aligned}
$$

which yields

$$
\begin{aligned}
A_{\Omega} & =\frac{1}{1-(a \cdot n)^{2}}\left\{2 J_{\Omega}^{a}-J_{\Omega}\right\} \\
B_{\Omega} & =J_{\Omega}-J_{\Omega}^{a}
\end{aligned}
$$

in terms of the functions $J_{\Omega}$ and $J_{\Omega}^{a}$ which can easily be calculated (note that $J_{\Omega}$ is the reciprocal of Colsher's filter (30)). Using this result, and following the same lines as for the full acceptance case, we get for the Fourier transform of the backprojected directional data

$$
\begin{aligned}
\|\nu\| \hat{b}_{i}^{1}(\nu) & =\hat{v}_{j}(\nu) \hat{k}_{i, j}^{1}\left(\frac{\nu}{\|\nu\|}\right) \\
& =A_{\Omega}\left({ }^{s} \hat{v}_{j}(\nu) a_{j}\right)\left(a_{i}-(a \cdot n) n_{i}\right)+B_{\Omega}{ }^{s} \hat{v}_{i}(\nu)
\end{aligned}
$$


The solenoid part of the vector field is then reconstructed as

$$
{ }^{s} \hat{v}_{j}(\nu)=\frac{\|\nu\| \hat{b}_{i}^{1}(\nu)}{B_{\Omega}}\left\{\delta_{i, j}-\frac{A_{\Omega}}{J_{\Omega}^{a}}\left(a_{j}-(n \cdot a) n_{j}\right) a_{i}\right\},
$$

provided the denominators do not vanish. This only happens when $\Omega$ reduces to a single equatorial circle normal to $a$, since then $J_{\Omega}^{a}(a)=0$. In the other limit where $\Omega \rightarrow S^{2}$, one has $J_{S^{2}}=2 \pi$ and $J_{S^{2}}^{a}=\pi$ so that $A_{S^{2}}=0$ and $B_{S^{2}}=\pi$. Equation (63) reduces then to (49).

Note finally that the case where $\Omega$ is the union of two equatorial circles can be analyzed following the same lines as above for each great circle and then adding the two contributions. One then checks Sparr's result [2] that the solenoid part can be reconstructed from such data.

\subsection{The tensor case with limited acceptance: equatorial band}

Following the same lines as for vectors, we extend the analysis in section 5.3 to the case where $\Omega$ is an equatorial band with axis $a \in S^{2}$. We first calculate the tensor (18), which may now be built not only from $\delta_{i, j}$ and $n_{j}$ but also from the axis $a$. Taking into account the full symmetry of the tensor and the fact that it must be divergence free, its most general form is:

$$
\begin{aligned}
k_{i, j, k, l}^{1,1}(n) & =C_{\Omega}(n)\left\{\epsilon_{i, j} \epsilon_{k, l}+\epsilon_{i, k} \epsilon_{j, l}+\epsilon_{i, l} \epsilon_{j, k}\right\} \\
& +\frac{D_{\Omega}(n)}{1-(a \cdot n)^{2}}\left\{\epsilon_{i, j} \stackrel{\circ}{a}_{k} \stackrel{\circ}{a}_{l}+\epsilon_{i, k} \stackrel{\circ}{a}_{j} \stackrel{\circ}{a}_{l}+\epsilon_{i, l} \stackrel{\circ}{a}_{k} \stackrel{\circ}{a}_{j}+\epsilon_{k, l} \stackrel{\circ}{a}_{i} \stackrel{\circ}{a}_{j}+\epsilon_{j, l} \stackrel{\circ}{a}_{k} \stackrel{\circ}{a}_{i}+\epsilon_{j, k} \stackrel{\circ}{a}_{i} \stackrel{\circ}{a}_{l}\right\} \\
& +\frac{E_{\Omega}(n)}{\left(1-(a \cdot n)^{2}\right)^{2}} \stackrel{\circ}{i}_{i} \stackrel{\circ}{a}_{j} \stackrel{\circ}{a}_{k} \stackrel{\circ}{a}_{l},
\end{aligned}
$$

where $\epsilon_{i, j}$ (defined above, see above eq. (50)) and $\stackrel{\circ}{a}_{i}=a_{i}-(a \cdot n) n_{i}$ are both divergence free. To determine the scalars $C, D$ and $E$, we calculate contractions of the tensor, namely:

$$
\begin{aligned}
k_{i, i, k, k}^{1,1} & =J_{\Omega}(n)=8 C_{\Omega}+8 D_{\Omega}+E_{\Omega} \\
\frac{k_{i, i, k, l}^{1,1} a_{k} a_{l}}{1-(a \cdot n)^{2}} & =J_{\Omega}^{a}(n)=4 C_{\Omega}+7 D_{\Omega}+E_{\Omega} \\
\frac{k_{i, j, k, l}^{1,1} a_{i} a_{j} a_{k} a_{l}}{\left(1-(a \cdot n)^{2}\right)^{2}} & =J_{\Omega}^{a a}(n)=3 C_{\Omega}+6 D_{\Omega}+E_{\Omega},
\end{aligned}
$$

where the two first $J$ functions are defined above in (60) and

$$
J_{\Omega}^{a a}(n) \equiv \frac{1}{\left(1-(a \cdot n)^{2}\right)^{2}} \int_{\Omega} d \theta(a \cdot \theta)^{4} \delta(n \cdot \theta)
$$

One then obtains:

$$
\begin{aligned}
C_{\Omega} & =\frac{1}{3}\left(J_{\Omega}-2 J_{\Omega}^{a}+J_{\Omega}^{a a}\right) \\
D_{\Omega} & =\frac{1}{3}\left(-J_{\Omega}+5 J_{\Omega}^{a}-4 J_{\Omega}^{a a}\right) \\
E_{\Omega} & =J_{\Omega}-8 J_{\Omega}^{a}+8 J_{\Omega}^{a a}
\end{aligned}
$$


Having now determined the tensor $k$, we calculate the Fourier transform of the backprojection of the directional measurement according to (17):

$$
\begin{aligned}
\|\nu\| \hat{b}_{i, j}^{1,1}(\nu) & =\hat{t}_{k, l}(\nu) \hat{k}_{i, j, k, l}^{1,1}\left(\frac{\nu}{\|\nu\|}\right)=C_{\Omega}\left\{\epsilon_{i, j} \hat{t}_{k, k}+2 \hat{t}_{i, j}\right\} \\
& +\frac{D_{\Omega}}{1-(a \cdot n)^{2}}\left\{\epsilon_{i, j}\left(\hat{t}_{k, l} a_{k} a_{l}\right)+2 \stackrel{\circ}{a}_{j} T_{i}+2 \stackrel{\circ}{a}_{i} T_{j}+\stackrel{\circ}{a}_{i} \stackrel{\circ}{a}_{j} \hat{t}_{k, k}\right\} \\
& +\frac{E_{\Omega}}{\left(1-(a \cdot n)^{2}\right)^{2}} \stackrel{\circ}{a}_{i} \stackrel{\circ}{a}_{j}\left(\hat{t}_{k, l} a_{k} a_{l}\right),
\end{aligned}
$$

where we have omitted the arguments $n=\nu /\|\nu\|$ and the ${ }^{s}$ notation for the solenoid part of the tensor, and have defined:

$$
T_{i} \equiv \hat{t}_{i, j} a_{j}
$$

This equation can be solved to recover the solenoid tensor $\hat{t}_{i, j}$ provided we can express $T_{i}, \hat{t}_{k, k}$ and $\hat{t}_{k, l} a_{k} a_{l}$ as a function of the data, i.e. of $\hat{b}_{i, j}^{1,1}$. This can be done by calculating contractions $\hat{b}_{k, k}^{1,1}, \hat{b}_{k, l}^{1,1} a_{k} a_{l}$ and $\hat{b}_{i, j}^{1,1} a_{j}$. After lengthy but straightforward calculations, one obtains:

$$
\begin{aligned}
\hat{t}_{i, j} & =\frac{\|\nu\|}{2 C_{\Omega}}\left\{\hat{b}_{i, j}^{1,1}+\frac{F_{1}}{1-(a \cdot n)^{2}}\left(\stackrel{\circ}{a}_{j}\left(\hat{b}_{i, k}^{1,1} a_{k}\right)+\stackrel{\circ}{a}_{i}\left(\hat{b}_{j, k}^{1,1} a_{k}\right)\right)+F_{2} \epsilon_{i, j} \hat{b}_{k, k}^{1,1}\right. \\
& \left.+\frac{F_{3}}{1-(a \cdot n)^{2}}\left\{\epsilon_{i, j} \hat{b}_{k, l}^{1,1} a_{k} a_{l}+\stackrel{\circ}{a}_{i} \stackrel{\circ}{j}_{j} \hat{b}_{k, k}^{1,1}\right\}+\frac{F_{4}}{\left(1-(a \cdot n)^{2}\right)^{2}} \stackrel{\circ}{a}_{i} \stackrel{\circ}{a}_{j} \hat{b}_{k, l}^{1,1} a_{k} a_{l}\right\},
\end{aligned}
$$

where the scalars are given by:

$$
\begin{aligned}
& F_{1}=\frac{J_{\Omega}-5 J_{\Omega}^{a}+4 J_{\Omega}^{a a}}{3\left(J_{\Omega}^{a}-J_{\Omega}^{a a}\right)} \\
& F_{2}=\frac{-J_{\Omega} J_{\Omega}^{a a}+3\left(J_{\Omega}^{a}\right)^{2}-4 J_{\Omega}^{a} J_{\Omega}^{a a}+2\left(J_{\Omega}^{a a}\right)^{2}}{3\left(J_{\Omega} J_{\Omega}^{a a}-\left(J_{\Omega}^{a}\right)^{2}\right)} \\
& F_{3}=\frac{3 J_{\Omega} J_{\Omega}^{a a}+\left(J_{\Omega}^{a}\right)^{2}-2 J_{\Omega}^{a} J_{\Omega}^{a a}-2 J_{\Omega} J_{\Omega}^{a a}}{3\left(J_{\Omega} J_{\Omega}^{a}-\left(J_{\Omega}^{a}\right)^{2}\right)} \\
& F_{4}=\frac{2\left(J_{\Omega}-2 J_{\Omega}^{a}+J_{\Omega}^{a a}\right)\left(J_{\Omega}^{a}\left(J_{\Omega}+J_{\Omega}^{a}\right)-2 J_{\Omega} J_{\Omega}^{a a}\right)}{3\left(J_{\Omega}^{a}-J_{\Omega}^{a a}\right)\left(J_{\Omega} J_{\Omega}^{a a}-\left(J_{\Omega}^{a}\right)^{2}\right)} .
\end{aligned}
$$

If $\Omega$ is an equatorial band not limited to a single circle, one can easily show that all denominators appearing above, do not vanish. Indeed,

$$
C_{\Omega}(n)=\frac{1}{3} \int_{\Omega} d u \delta(u \cdot n) \frac{\left(1-(a \cdot n)^{2}-(a \cdot u)^{2}\right)^{2}}{\left(1-(a \cdot n)^{2}\right)^{2}} .
$$

Note first that there is no singularity in $n=a$ because the denominator is always larger than or equal to the numerator. Next, note that this integral vanishes only if $a$ is always in the plane subtended by $u$ and $n$ and this cannot possibly be true for all $u \in \Omega$ such that $u \cdot n=0$, unless when $a=n$ but in that case the denominator vanishes and the integral has a finite non zero limit. Thus, $C_{\Omega}(n)>0$. Similarly, one can prove that $J_{\Omega}^{a}-J_{\Omega}^{a a}>0$ and $J_{\Omega} J_{\Omega}^{a a}-\left(J_{\Omega}^{a}\right)^{2}>0$. 


\subsection{The tensor case with limited acceptance: three great circles}

We extend the analysis in section 5.3 to the case where $\Omega$ is the union of three great circles orthogonal to three linearly independent unit vectors $a^{r}, r=1,2,3$ :

$$
\Omega=\cup_{r=1}^{3} \Omega^{r} \quad \text { with } \quad \Omega^{r}=\left\{\theta \mid \theta \cdot \theta=1, \theta \cdot a^{r}=0\right\} .
$$

We define the vectors $b_{p}^{r}=1 / 2 \epsilon_{r, r^{\prime}, r^{\prime \prime}} \epsilon_{p, i, k} a_{i}^{r^{\prime}} a_{k}^{r^{\prime \prime}}$, that is, $b^{1}=a^{2} \times a^{3}, b^{2}=a^{3} \times a^{1}, b^{3}=a^{1} \times$ $a^{2}$. We consider a fixed frequency $\nu$ vector, and define as before the unit vector $n=\nu /\|\nu\|$. We also define as before the orthogonal projections of the vectors $a^{r}$ onto the plane $\nu^{\perp}$, $\stackrel{\circ}{a}_{i}^{r}=a_{i}^{r}-\left(a^{r} \cdot n\right) n_{i}$. These three vectors are divergence-free.

We start from the directional measurement $p^{11}$ of the X-ray transform of the tensor field $t_{i, j}(x)$, as defined in equation (6). Using the central section theorem, equation (11), we can access the given frequency $\nu$ using the X-ray transform along one direction in each great circle. These three directions are

$$
\theta^{r}(\nu)=\frac{a^{r} \times n}{\sqrt{c^{r}(n)}} \in \Omega^{r} \quad r=1,2,3,
$$

with $c^{r}(n)=\left\|a^{r} \times n\right\|^{2}=1-\left(a^{r} \cdot n\right)^{2}$ and we assume that the frequency is not along one of the three great circle axis, i.e. we assume that $c^{r}(n) \neq 0, r=1,2,3$. This is also written component wise as

$$
\theta_{i}^{r}(\nu)=\frac{1}{\sqrt{c^{r}(n)}} \epsilon_{i, p, j} a_{p}^{r} n_{j} .
$$

Then, we have the central section theorem, equation (11),

$$
\hat{p}^{1,1}\left(\nu, \theta^{r}(\nu)\right)=\theta_{i}^{r}(\nu){ }^{s} \hat{t}_{i, j}(\nu) \theta_{j}^{r}(\nu) \quad r=1,2,3,
$$

where we indicate that the $p^{1,1}$ only feels the solenoidal component of the tensor field.

Our problem is now to recover the solenoidal part of the tensor from the directional measurement $p^{1,1}$ on the three great circles. The solution is unique for all frequencies such that $\nu \cdot b^{r} \neq 0$ for $r=1,2,3$, and can be written as:

$$
\begin{aligned}
{ }^{s} \hat{t}_{i, j}(\nu) & =\frac{-c^{1}(n)}{2\left(n \cdot b^{2}\right)\left(n \cdot b^{3}\right)}\left(\stackrel{\circ}{a}_{i}^{2} \stackrel{a}{a}_{j}^{3}+\stackrel{\circ}{a}_{i}^{3} \stackrel{\circ}{a}_{j}^{2}\right) \hat{p}^{1,1}\left(\nu, \theta^{1}(\nu)\right) \\
& +\frac{-c^{2}(n)}{2\left(n \cdot b^{1}\right)\left(n \cdot b^{3}\right)}\left(\stackrel{\circ}{a}_{i}^{1} \stackrel{a}{a}_{j}^{3}+\stackrel{\circ}{a}_{i}^{3} \stackrel{a}{a}_{j}^{1}\right) \hat{p}^{1,1}\left(\nu, \theta^{2}(\nu)\right) \\
& +\frac{-c^{3}(n)}{2\left(n \cdot b^{1}\right)\left(n \cdot b^{2}\right)}\left(\stackrel{\circ}{a}_{i}^{2} \stackrel{a}{a}_{j}^{1}+\stackrel{\circ}{a}_{i}^{1} \stackrel{\circ}{a}_{j}^{2}\right) \hat{p}^{1,1}\left(\nu, \theta^{3}(\nu)\right) .
\end{aligned}
$$

To prove this result, first note that the tensor (77) is solenoidal because $n_{i} \stackrel{\circ}{a}_{i}^{r}=0$ for each $r=1,2,3$. Next, note that replacing the tensor ${ }^{s} \hat{t}_{i, j}$ in equation (76) by $\stackrel{\circ}{a}_{i}^{2} \stackrel{\circ}{a}_{j}^{3}+\stackrel{\circ}{a}_{i}^{3} \stackrel{\circ}{a}_{i}^{2}$ yields,

$$
\begin{aligned}
A^{r}:=\theta_{i}^{r}\left(\stackrel{\circ}{a}_{i}^{2} \stackrel{a}{a}_{j}^{3}+\stackrel{\circ}{a}_{i}^{3} \stackrel{a}{a}_{i}^{2}\right) \theta_{j}^{r} & =\theta_{i}^{r}\left(a_{i}^{2} a_{j}^{3}+a_{i}^{3} a_{j}^{2}\right) \theta_{j}^{r} \\
& =\frac{1}{c^{r}(n)} \epsilon_{i, p, q} a_{p}^{r} n_{q}\left(a_{i}^{2} a_{j}^{3}+a_{i}^{3} a_{j}^{2}\right) \epsilon_{j, k, l} a_{k}^{r} n_{l} .
\end{aligned}
$$


Due to the anti-symmetry of the $\epsilon, \epsilon_{i, p, q} a_{p}^{r} a_{i}^{2}=0$ if $r=2$, and hence, $A^{2}=A^{3}=0$ and $A^{1}=-2\left(n \cdot b^{2}\right)\left(n \cdot b^{3}\right) / c^{1}(n)$. Therefore, the tensor (77) is symmetrical, solenoidal and satisfies equation (76) for $r=1,2,3$. This concludes the proof.

Finally we need to discuss the special case of a frequency $\nu$ which is orthogonal to one of the vectors $b^{r}$, say $\nu \cdot b^{1}=0$. As $b^{1}=a^{2} \times a^{3}$, this means that $n$ is in the plane spanned by $a^{2}$ and $a^{3}$. Then, the vectors $a^{2} \times n$ and $a^{3} \times n$ are colinear, hence $\theta^{2}(\nu)= \pm \theta^{3}(\nu)$. In this case, $\hat{p}^{1,1}\left(\nu, \theta^{2}(\nu)\right)=\hat{p}^{1,1}\left(\nu, \theta^{3}(\nu)\right)$, and therefore we get only two equations instead of three to determine the solenoidal tensor. The solution is not unique in this case. The only exception is if $\nu$ is along $a^{2}$ (or along $a^{3}$ ) because in that case we can access this frequency from any $\theta \in \Omega^{2}\left(\Omega^{3}\right)$.

\subsection{Reconstruction from a single directional measurement of the Radon transform}

In the previous sections we have seen that

1. The potential components of vectors (tensors) are in the null space of the directional measurement $p^{1}\left(p^{1,1}\right)$ of the 3D X-ray transform,

2. The solenoid part can be recovered from this measurement not only when $\Omega=S^{2}$ but even with a smaller set of measured parallel projections.

We now consider the corresponding measurement of the 3D Radon transform, i.e. $r^{1}$ or $r^{1,1}$ for tensors, i.e. we measure plane integrals of the scalar product of the vector or tensor with the vector $\theta$ normal to the plane of integration. The information which can be extracted from such data is elicited by noting that the Fourier transform of the corresponding directional backprojection are given by (see equation 23 and 24):

$$
\hat{b}_{i}^{1}(\nu)=\frac{2}{\|\nu\|^{2}} n_{i} n_{j} \hat{v}_{j}(\nu)
$$

or

$$
\hat{b}_{i, j}^{\alpha, \beta}(\nu)=\frac{2}{\|\nu\|^{2}} n_{i} n_{j} n_{k} n_{l} \hat{t}_{k, l}(\nu),
$$

with $n=\nu /\|\nu\|$. Comparing this with the Fourier description of the decomposition into solenoid and potential components we see that

1. The potential part of vector fields can be recovered,

2. But the potential part of tensors cannot be recovered.

For example, a tensor field such that its Fourier transform has the form

$$
\hat{t}_{i, j}(\nu)=f(\nu)\left\{e_{i}^{1}(\nu /\|\nu\|) e_{j}^{2}(\nu /\|\nu\|)+e_{j}^{1}(\nu /\|\nu\|) e_{i}^{2}(\nu /\|\nu\|)\right\},
$$

where $e^{1}(n)=n$ and $e^{2}(n)$ is normal to $n$, is transparent both to the directional X-ray transform $p^{1,1}$ and to the directional Radon transform $r^{1,1}$. Other directional data are therefore required to reconstruct the whole tensor field, whereas for vectors $p^{1}$ and $r^{1}$ are sufficient, as shown by Sparr [2]. 


\section{Summary}

Here we have developed formulations for the reconstruction of 3D tensor fields from planar (Radon) and line-integral (X-ray) projections of 3D vector and tensor fields. Much of the motivation for this work is the potential application of MRI to perform diffusion tensor tomography. The goal is to develop a theory for the reconstruction of both Radon planar and X-ray or line-integral projections because of the flexibility of MRI to obtain both of these type of projections in 3D. The development presented here for the linear tensor tomography problem provides insight into the structure of the nonlinear MRI diffusion tensor inverse problem.

A particular application of tensor imaging in MRI is the potential application of cardiac diffusion tensor tomography for determining in vivo cardiac fiber structure. One difficulty in the cardiac application is the motion of the heart. This presents a need for developing future theory for tensor tomography in a motion field. This means developing a better understanding of the MRI signal for diffusion processes in a deforming media.

The techniques developed may allow the application of MRI tensor tomography for the study of structure of fiber tracts in the brain, atherosclerotic plaque, and spine in addition to fiber structure in the heart. However, the relations presented are also applicable to other fields in medical imaging such as diffraction tomography using ultrasound. The mathematics presented can also be extended to exponential Radon transform of tensor fields and to other geometric acquisitions such as cone beam tomography of tensor fields.

\section{Acknowledgements}

This work was supported by the Benning Trust Funds and by the Director, Office of Energy Research, Office of Biological and Environmental Research, Medical Applications and Biophysical Research Division of the U.S. Department of Energy under contract DE-AC03$76 \mathrm{SF} 00098$.

\section{References}

[1] Mathematics and Physics of Emerging Biomedical Imaging. National Academy Press, Washington, D.C., 1996.

[2] G Sparr and K Strahlen. Vector Field Tomography - An Overview. Technical report, Lund University, Lund Institute of Technology: Center for Mathematical Sciences, Sweden, 1998.

[3] J M Guccione, A D McCulloch, and L K Waldman. Passive material properties of intact ventricular myocardium determined from a cylindrical model. Trans of the ASME, 113:42-55, 1991.

[4] P S Chen, Y M Cha, B B Peters, and L S Chen. Effects of myocardial fiber orientation on the electrical induction of ventricular fibrillation. Am J Physiol, 264 (6 Pt2):H17601773, 1993. 
[5] E Debreuve and G T Gullberg. Dense electrical map reconstruction from ECG/MCG measurements using a priori myocardial fiber structure and activation sequence information. In Proceedings of the 23rd Annual International Conference of the IEEE Engineering in Medicine and Biology Society, Istanbul, Turkey, October, 2001.

[6] G Stroink, W Moshage, and S Achenbach. Magnetism in Medicine: A Handbook, chapter Cardiomagnetism, pages 136-189. Wiley-VCH, New York, 1998.

[7] S A Johnson, J F Greenleaf, M Tanaka, and G Flandro. Acoustical Holography, volume 7, chapter Reconstructing three-dimensional fluid velocity vector fields from acoustic transmission measurements, pages 307-326. Plenum, New York, 1977.

[8] S E Serge. The measurement of poloidal magnetic field in a tokomak by the change of polarization of an electromagnetic wave. Plasma Phys, 20:295-307, 1978.

[9] W Munk and C Wunsch. Ocean acoustic tomography: A scheme for large-scale monitoring. Deep-Sea Research, 26A:123-161, 1979.

[10] D M Kramer and P C Lauterbur. On the problem of reconstructing images of nonscalar parameters from projections. Application to vector fields. IEEE Trans Nucl Sci, NS26:2674-2677, 1979.

[11] S Takuso et al. Introduction of mass conversations law to improve the tomographic estimation of flow velocity distribution from differential time-of-flight data. Acoust Soc Amer, 77:2104-2106, 1985.

[12] H M Hertz. Kerr effect tomography for nonintrusive spatially resolved measurements of asymmetric electric field distributions. Applied Optics, 25:914-921, 1986.

[13] H K Aben. Kerr effect tomography for general axisymmetric field. Applied Optics, 26:2921-2924, 1987.

[14] B M Howe, P F Worchester, and W Munk. Ocean acoustic tomography: Mesoscale velocity. J Geophys Research, 92:3785-3805, 1987.

[15] G W Faris and R L Byer. Three-dimensional beam-deflection optical tomography of a supersonic jet. Applied Optics, 27:5202-5212, 1988.

[16] S J Norton. Tomographic reconstruction of 2-D vector fields: Application to flow imaging. Geophys J, 97:161-168, 1988.

[17] Y Chen, Y Jia, and W Shu. Utilizing phase retardation integration method of flow birefringence to analyze the flow with symmetric plane. Acta Mech Sin, 5:374-381, 1990.

[18] K B Winters and D Rouseff. A filtered backprojection method for the tomographic reconstruction of fluid vorticity. Inverse Problems, 6:L33-L38, 1990. 
[19] D Rouseff, K B Winters, and T E Ewart. Reconstruction of oceanic microstructure by tomography: A numerical feasibility study. J Geophysical Research, 96:8823-8833, 1991.

[20] H Braun and A Hauck. Tomographic reconstruction of vector fields. IEEE Trans on Signal Process, 39:464-471, 1991.

[21] S J Norton. Unique tomographic reconstruction of vector fields using boundary data. IEEE Trans Image Processing, 1:406-412, 1992.

[22] P Juhlin. Principles of Doppler Tomography. Technical report, Lund University, Lund Institute of Technology: Center for Mathematical Sciences, Sweden, 1992.

[23] K B Winters and D Rouseff. Tomographic reconstruction of stratified fluid flow. IEEE Trans. on Ultrasonics, Ferroelectrics and Frequency Control, 40:26-33, 1993.

[24] P Juhlin. Doppler tomography. In Proceedings of the 15th Annual International Conference of the IEEE Engineering in Medicine and Biology Society, pages 212-213, 1993.

[25] M Zahn. Transform relationship between Kerr-effect optical phase shift and non-uniform electrical field distributions. Trans on Dielectrics and Electrical Insulation, 1:235-246, 1994.

[26] A Schwarz. Three-dimensional reconstruction of temperature and velocity fields in a furnace. In Proceeding of ECAPT, The European Concerted Action on Process Tomography, pages 227-233, 1994.

[27] A J H Donne. High spatial resolution interferometry and polarimetry in hot plasmas. Rev Sci Instrum, 66:3407-3423, 1995.

[28] N Efremov, N Pamentov, and V Kharachenko. Tomography of ion and atom velocities in plasmas. J Quant Spectrosc Radiation Transfer, 6:723-728, 1995.

[29] A M Denisov, A A Popov, and V V Sterlyadkin. Doppler tomography problem for a two-dimensional vector field. Moscow University Computational Mathematics and Cybernetics, 1:17-20, 1995.

[30] L Desbat and A Wernsdorfer. Direct algebraic reconstruction and optimal sampling in vector field tomography. IEEE Trans on Signal Processing, 43:1798-1808, 1995.

[31] L Desbat. Efficient parallel sampling in vector field tomography. Inverse Problems, 11:995-1003, 1995.

[32] G Sparr, K Strahlen, K Lindstrom, and H W Persson. Doppler tomography for vector fields. Inverse Problems, 11:1051-1061, 1995.

[33] K Strahlen. Some Integral Transforms of Vector Fields. Licentiate thesis, Lund University, Department of Mathematics, Sweden, 1996. 
[34] J L Prince. Convolution backprojection formulas for 3-D vector tomography with application to MRI. IEEE Trans on Image Processing, 5:1462-1472, 1996.

[35] N F Osman and J L Prince. 3D vector tomography on bounded domains. Inverse Problems, 14:185-196, 1998.

[36] H Aben. Integrated Photoelasticity. McGraw-Hill, Inc., New York, 1979.

[37] H K Aben, J I Josepon, and K-J E Kell. The case of weak birefringence in integrated photoelasticity. Opt Lasers Eng, 11:145-157, 1989.

[38] V A Sharafutdinov. Tomographic problem of photoelasticity. In G Levin, editor, Proceedings of SPIE: Analytical Method for Optical Tomography, pages 234-243, Zvenigorod, Russia, 1991.

[39] K-J Kell and A Puro. The case of very weak birefringence in the method of integrated photoelasticity. Opt Spectrosk, 70:390-393, 1991.

[40] A E Puro. Investigation of the stress state of elastic models by the method of optical tomography. Int Appl Mech, 28:173-177, 1992.

[41] Y A Andrienko, M S Duboikov, and A D Gladun. Optical tensor field tomography: the Kerr effect and axisymmetric integrated photoelasticity. J Opt Soc Am A, 9:1765-1768, 1992.

[42] Y A Andrienko and M S Dubovikov. Optical tomography of tensor fields: The general case. J Opt Soc Am A, 11:1626-1631, 1994.

[43] H Aben and A Puro. Photoelastic tomography for three-dimensional flow birefringence studies. Inverse Problems, 13:215-221, 1995.

[44] A E Puro. On the tomographic method in magnetophotoelasticity. Opt Spectrosk, 81:119-125, 1996.

[45] D Schupp. Determination of 3-D stress by optical tensor field tomography. In Proceedings of the SPIE - The International Society for Optical Engineering, pages 431-441, Munich, Germany, June 16-19, 1997.

[46] A Puro. Magnetophotoelasticity as parametric tensor field tomography. Inverse Problems, 14:1315-1330, 1998.

[47] S Amarnath and K R Y Simha. Tomographic data acquisition in axisymmetric specimens. Experimental Techniques, 22:21-25, 1998.

[48] D Schupp. Determination of the 3D stress tensor in photoelastic materials by optical tensor field tomography. In Proceedings of the SPIE - The International Society for Optical Engineering, pages 56-67, Denver, CO, July 21-23, 1999.

[49] A Puro. Cormack-type inversion of exponential radon. Inverse Problems, 17:179-188, 2001. 
[50] A E Puro. Parametric tomography of internal stresses. Optika i Spektroskopiya, 90:664$674,2001$.

[51] V A Sharafutdinov. Integral Geometry of Tensor Fields. V O Science, Novosibirsk, 1993.

[52] G T Gullberg, D Ghosh Roy, G L Zeng, A L Alexander, and D L Parker. Tensor tomography. IEEE Trans on Nucl Sci, 46:991-1000, 1999.

[53] G T Gullberg and M Defrise. Three-dimensional tomography for vector and tensor fields. In F Beekman, M Defrise, and M Viergever, editors, Proceedings of the 1999 International Meeting on Fully Three-Dimensional Image Reconstruction in Radiology and Nuclear Medicine, pages 369-372, Egmond aan Zee, The Netherlands, June 23-26, 1999.

[54] G T Gullberg, M Defrise, V Panin, and G L Zeng. Backprojection filtering algorithms for reconstruction of vector and second order tensor fields. In 2000 IEEE Nuclear Science Symposium and Medical Imaging Conference Record, pages 15-277-15-281, Lyon, France, October 15-20, 2000.

[55] V Panin, G L Zeng, and G T Gullberg. An iterative approach to tensor tomography. In 2000 IEEE Nuclear Science Symposium and Medical Imaging Conference Record, pages 15-272-15-276, Lyon, France, October 15-20, 2000.

[56] G T Gullberg, M Defrise, V Panin, and G L Zeng. Efficient cardiac diffusion tensor MRI by three-dimensional reconstruction of solenoidal tensor fields. Magnetic Resonance Imaging, 19:233-256, 2001.

[57] V Y Panin, G L Zeng, G T Gullberg, A L Alexander, and D L Parker. An iterative regularized algorithm for tensor tomography in MRI. In Proceedings of the International Society for Magnetic Resonance in Medicine, 9th Scientific Meeting and Exhibition, page 765, Glasgow, Scotland, April 21-27, 2001.

[58] V Y Panin, G L Zeng, and G T Gullberg. Regularized iterative reconstruction in tensor tomography using gradient constraints. IEEE Trans on Nucl Sci, 49:2387-2393, 2002.

[59] V Y Panin, G L Zeng, M Defrise, and G T Gullberg. Diffusion tensor MR imging of principal directions: A tensor tomography approach. Phys Med Biol, 47:2737-2757, 2002.

[60] A B Cheryauka, J N Lee, A A Samsonov, M Defrise, and G T Gullberg. MRI diffusion tensor reconstruction with PROPELLER data acquisition. Magn Reson Imag, 22:139$148,2004$.

[61] D Rouseff and K B Winters. Two-dimensional vector flow inversion by diffraction tomography. Inverse Problems, 10:687-697, 1994.

[62] P M Morse and U Ingaard. Theoretical Acoustics. McGraw-Hill, New York, 1968. 
[63] I M Romanishin. Acoustoelastic tomographic reconstruction of the tensor stress field. Defektoskopiya, 31:46-50, 1995.

[64] A S Kravchuk. Tomographic algorithms in the theory of elasticity. Prikladnaya Matematika i Mekhanika, 63:509-512, 1992.

[65] Iu E Anikonov. Inverse Problems for Kinetic and Other Evolution Equations. Utrecht, The Netherlands, 2001.

[66] D C Montgomery. Plasma Kinetic Theory. McGraw-Hill, New York, 1964.

[67] S Y Berezhna, I V Berezhnyi, O M Krupych, and O G Vlokh. Some peculiarities of dielectric tensor field optical tomography technique. In Proceedings of the SPIE - The International Society for Optical Engineering, pages 622-627, Kiev, Ukraine, May 11-13, 19951982.

[68] H Kubo. Approach to dielectric tensor slice-type tomography. J Opt Soc Am A, 14:22992313, 1997.

[69] W Huatao and J M Lees. Cartesian parametrization of anisotropic traveltime tomography. Geophys J Int, 137:64-80, 1999.

[70] Badurek G Hochhold M, Leeb H. Neutron spin tomography: a tool to visualize magnetic domains in bulk materials. J Phys Soc Jpn, 65:292-295, 1996.

[71] S Coen. Gravitational delay time tomography with photons in a stationary universe. Classical and Quantum Gravity, 18:L53-L59, 2001.

[72] I M Gelfand, S G Gindikin, and M I Graev. Integral geometry in affine and projective spaces. J Sov Math, 18:39-167, 1982.

[73] J Colsher. Fully-three-dimensional positron emission tomography. Phys Med Biol, 25:103-115, 1980.

[74] S S Orlov. Theory of three-dimensional reconstruction. 1. Conditions of a complete set of projections. Sov Phys Crystallography, 20:321, 1976. 